\title{
Regressão linear e quadrática: Análises comparativas de efeito em medidas do comportamento organizacional
}

Maria Isabel de Campos. Universidade São Francisco

Fabián J. M. Rueda. Universidade São Francisco

\section{Resumo}

Este estudo apresenta análises comparativas entre modelos de regressão linear múltipla e de regressão quadrática. Foram utilizados valores organizacionais reais e desejados como variáveis independentes e satisfação com o trabalho como variável dependente. A amostra contou com 213 trabalhadores de todas as regiões do Brasil, homens e mulheres, predominantemente com formação em nível superior, atuantes em empresas de diversos setores. A regressão quadrática possibilitou o encontro de explicações de variância de maior magnitude, quando comparada ao modelo linear. Análises de gráficos de superfície ampliaram o entendimento a respeito dos efeitos promovidos pelas variáveis independentes sobre as dependentes. São apresentadas considerações sobre a seleção de modelos de regressão tanto no contexto acadêmico, quanto na prática organizacional.

Palavras-chave: regressão estatística; compatibilidade indivíduo-organização; comportamento organizacional.

\begin{abstract}
Linear and quadratic regression: Comparative analysis of effect on organizational behavior measures. This study presents comparative analysis of multiple linear regression model and quadratic regression. Organizational values (actual and desired) were used as independent variables and job satisfaction as the dependent variable. The sample consisted of 213 workers from all regions of Brazil, men and women, mostly with higher education, working in various industries. The quadratic regression resulted in variance explanations of greater magnitude when compared to the linear model. Surface analysis charts expanded the understanding of the effects promoted by the independent variables on the dependent. Considerations regarding the selection of regression models in the academic context and in organizational practice are provided.
\end{abstract}

Keywords: statistical regression; personal-organization fit; organizational behavior.

\section{Resumen}

Regresión lineal y cuadrática: Análisis comparativo del efecto de las medidas de comportamiento organizacional. Este estudio presenta un análisis comparativo de los modelos de regresión lineal múltiples y la regresión cuadrática. Se utilizaron los valores organizacionales (reales y deseados) como variables independientes y la satisfacción en el trabajo como la variable dependiente. La muestra fue de 213 trabajadores de todas las regiones de Brasil, hombres y mujeres, en su mayoría con la educación superior, trabajando en diversos sectores. La regresión cuadrática resultó en explicaciones de varianza de mayor magnitud en comparación con lo modelo lineal. Gráficos de análisis de superficie ampliaron la comprensión de los efectos promovidos por las variables independientes sobre las dependientes. Se proporcionan consideraciones relativas a la selección de modelos de regresión en el contexto académico como en la práctica de la organización.

Palabras clave: regresión estadística; compatibilidad individuo-organización; conducta organizacional. 
A Psicologia Organizacional e do Trabalho (PO\&T) é entendida como uma subárea de conhecimento da Psicologia e como um campo de aplicação de conhecimentos ou de intervenção. Tem o fazer humano como objeto de estudo e os contextos do trabalho e das organizações como locais desse estudo, no primeiro papel. No segundo, é requerido dela que dê respostas a questões práticas, no que diz respeito a interações entre o comportamento humano, o trabalho e as organizações. Assim, a PO\&T passa por processos de desenvolvimento relacionados à pesquisa científica e à atuação na prática organizacional, de forma que pesquisa e prática precisam, periodicamente, estabelecer contatos que permitam que produção e utilização do conhecimento caminhem mais ou menos juntas e que benefícios possam emergir para todos os envolvidos (Borges-Andrade $\&$ Pagotto, 2010).

De acordo com Borges-Andrade e Pagotto (2010), a partir da década de 1970 revisões de literatura sobre o estudo do fazer humano passaram a receber o nome de Comportamento Organizacional (CO). O CO nasceu há cerca de cinco décadas como disciplina independente, oriunda da convergência de conhecimento entre áreas estabelecidas, como Psicologia, Economia e Sociologia. Atualmente, construtos comportamentais sob o foco do CO são considerados como uma base para a compreensão de resultados econômicos organizacionais, incluindo a vantagem competitiva e a lucratividade (Floyd \& Sputtek, 2011). Assim, é importante para a PO\&T, como campo de aplicação ou intervenção, que métodos adequados e precisos de medir o comportamento organizacional sejam criados e difundidos.

Essa importância é reconhecida em ambientes organizacionais, onde resultados de medidas são normalmente denominados de indicadores de desempenho. A Fundação Nacional da Qualidade (FNQ) define um indicador de desempenho como um dado numérico a que se atribui uma meta e que é trazido, periodicamente, à atenção dos gestores de uma organização. Esses indicadores conectam as estratégias ao resultado das atividades e devem atender às seguintes características: ser de fácil visibilidade, possibilitar uma visão balanceada do desempenho da organização, facilitar o entendimento dos direcionadores do negócio, e suportar a tomada de decisões visando influenciar os ambientes interno e externo à organização (FNQ, 2008). Dado o contexto, tais características são úteis também para orientar o desenvolvimento e a análise de Medidas do Comportamento Organizacional (MCO).
Uma das teorias que tem sido amplamente pesquisadas em MCO é a da compatibilidade, ou fit, entre indivíduo-organização (personal-organization fit - POF). Em essência, as pesquisas em POF preocupam-se com os antecedentes e as consequências da compatibilidade entre as pessoas e as organizações em que elas trabalham. Essa compatibilidade ocorre quando pelo menos uma entidade provê o que a outra precisa e/ou ambas compartilham características similares fundamentais (Kristoff, 1996) e sua existência promove resultados positivos para indivíduos e para as organizações. Entre eles destacam-se maior satisfação, ajustamento, comprometimento, desempenho, menores níveis de estresse e baixa intenção de sair da organização (Porto, Tamayo, \& Paschoal, 2012).

Estudos de POF foram realizados ao longo de décadas, com base em cálculos de diferenças de pontuação (difference scores), o que levou Edwards (1994a, 1994b) a sustentar um debate a respeito da qualidade dos resultados obtidos naquelas pesquisas, fossem as diferenças calculadas como algébricas, absolutas, quadráticas ou por outro meio. $O$ autor apresentou questionamentos com relação à confiabilidade e validade desses resultados. Suas publicações trouxeram um modelo alternativo a ser utilizado, orientando pesquisadores a aplicarem a regressão polinomial quadrática e a realizarem a análise de gráficos de superfície, ao invés de usarem regressão linear ( $R L$ ) na análise de POF. A solução proposta, regressão quadrática (RQ), possibilita, ainda segundo o autor, testar a forma da função que a diferença de pontuação pretende representar, ao invés de assumi-la como uma função linear.

Na polaridade contrária do debate encontraram-se autores como Tisak e Smith (1994), defendendo o uso das diferenças de pontuação e apresentando respostas e soluções às críticas apresentadas por Edwards. Com relação aos questionamentos a respeito da confiabilidade das medidas, Tisak e Smith (1994) afirmaram que as pesquisas em $\mathrm{CO}$ comumente utilizam medidas com múltiplos itens e fatores e que a confiabilidade, nesses casos, deve ser verificada por meio do coeficiente alfa, não podendo essas medidas serem consideradas como inerentemente não confiáveis.

Aos questionamentos a respeito da validade das medidas, que não poderiam ser interpretadas sem ambiguidade e que confundem o pesquisador (Edwards, 1994a), Tisak e Smith (1994) responderam que a análise deve ser feita pelos pesquisadores, caso a caso, e que as críticas indicam que as diferenças de pontuação podem nem sempre funcionar como esperado, mas que 
isso não justifica que seu uso seja abandonado a priori. Edwards (1994b, 2001) rebateu as defesas apresentadas por Tisak e Smith (1994), fortalecendo a posição da RQ como a solução que possibilita testar teorias e verificar seu real funcionamento, ao invés de assumi-las como corretas. Para Edwards (1994b) o uso de diferenças de pontuação pode promover eventuais ocorrências de enganos na validação dessas teorias.

Ocorre que desde a década de 1990, conforme Saraç, Efil e Eryilmaz (2014), estudiosos têm encontrado que a RQ pode aumentar a variância explicada em estudos de POF. Essa tem sido, conforme literatura da área, a escolha metodológica de diversos pesquisadores, entre eles De Haas e Van Eerde (2015), Lee e Antonakis (2014), Ramsey e van den Berk-Clark (2015) e, no Brasil, Sousa e Porto (2015).

Entretanto, a aplicação de RL em estudos que utilizam diferenças de pontuação pode ainda ser encontrada na literatura brasileira na última década, como é o caso em Borges, Argolo e Baker (2006). Ainda, novas escalas continuam a ser desenvolvidas adotando o cálculo de diferenças entre "o que é" (real) e "o que deveria ser" (desejado), como em Borges e Barros (2015), o que abre espaço para eventuais novos estudos em POF utilizando esse tipo de instrumento e diferenças de pontuação.

Em meio a esse histórico, uma pesquisa realizada pelos autores deste estudo em dezembro de 2015 na base de periódicos da Coordenação de Aperfeiçoamento de Ensino Superior (CAPES), no index-psi da Biblioteca Virtual em Saúde Psicologia (BVS) e no Google Acadêmico, usando os termos "regressão quadrática", "regressão polinomial" e "análise comparativa", não encontrou estudos brasileiros sobre análises de impacto em medidas do comportamento organizacional que aplicassem distintos modelos de regressão, possibilitando considerar, por meio de empirismo, as duas abordagens metodológicas que sustentaram o debate internacional no contexto de POF. Estudos nessa linha podem vir a contribuir para que os pesquisadores na área aprofundem o contato com os diferentes modelos e seus resultados comparados, facilitando tomadas de decisão não apenas na definição metodológica da aplicação, mas quiçá, no momento de desenho do projeto e definição da coleta a ser realizada.

Comparações desse cunho podem ser úteis também para profissionais da prática organizacional, que necessitam compreender as razões que levam acadêmicos a optar por um determinado modelo de análise e avaliar se ele é, de fato, o mais adequado ao seu cotidiano. Essa avalição deve considerar os recursos disponíveis (e.g. tempo, esforço, custo), em contraste com a necessidade de que as informações a serem apresentadas devem suportar o estabelecimento de índices que sejam não apenas precisos, mas também facilmente compreensíveis e monitoráveis, conforme os mencionados requisitos da FNQ (2008).

Este estudo teve, pelos motivos expostos, os objetivos de aplicar modelos de regressão linear múltipla e de regressão quadrática utilizando medidas do comportamento organizacional (valores organizacionais e satisfação no trabalho) e de realizar a comparação entre os resultados obtidos com os diferentes modelos de análise. Objetiva-se, ainda, apresentar considerações para que se reflita sobre a decisão de adotar, ou não, um determinado modelo, de forma a atender a necessidades da pesquisa acadêmica e da prática organizacional.

Como variável independente selecionou-se a compatibilidade (ou congruência) com os valores organizacionais, cujos resultados foram obtidos de três formas diferentes, com base nos escores de valores organizacionais (VO) reais e desejados. Como variável dependente utilizou-se a satisfação no trabalho (ST). A seleção dessas variáveis baseou-se nos seguintes critérios: 1) a operacionalização mais frequentemente utilizada em estudos de POF é a baseada em valores do indivíduo e da organização (Kristof, 1996); 2) a existência de uma escala desenvolvida por pesquisadores brasileiros para a mensuração de valores organizacionais que agrega o conceito de diferenças de pontuação entre os valores reais e desejados, adequada para o estudo de POF. Trata-se do Inventário de Valores Organizacionais - IVO (Tamayo, Mendes, \& Paz, 2000); 3) no entendimento de Tisak e Smith (1994) medidas multi-item e multidimensionais viabilizam melhores achados quando da aplicação de diferenças de pontuação colapsadas para a formação de um índice preditor, sendo este o caso das medidas realizadas com o IVO; 4) as medidas de satisfação no trabalho também são feitas por meio de escalas com múltiplos itens e fatores; 5) o IVO foi a escala aplicada por Borges et al. (2006), que utilizaram a regressão linear para analisar o efeito dos valores organizacionais sobre o burnout; 6) de acordo com Porto et al. (2012), estudos de meta-análise indicaram haver forte correlação de POF com a satisfação no trabalho (0,50); e 7) de acordo com Edwards (2008), as principais teorias a respeito de ST carregam, em sua essência, os conceitos de POF. 
Regressão linear e quadrática: Análises comparativas de efeito em medidas do comportamento organizacional

Elucida-se que os valores organizacionais são definidos como princípios ou crenças, organizados hierarquicamente, relativos a comportamentos ou metas organizacionais desejáveis, que orientam a vida da empresa e estão a serviço de interesses individuais, coletivos ou mistos (Tamayo \& Borges, 2006). Neste sentido, os VO podem ser considerados como uma variável de nível macro (Puente-Palacios \& Laros, 2009; Puente-Palacios, Porto, \& Martins, 2016), uma vez que refletem o contexto organizacional.

A satisfação no trabalho, por sua vez, representa o grau de contentamento do trabalhador com relação a distintas dimensões que compõem o seu labor, sendo as dimensões colegas, salário, chefia, promoções e natureza do trabalho as mais presentes na literatura das últimas décadas (Siqueira, 2008). A ST pode ser compreendida como uma variável de nível micro (Puente-Palacios \& Laros, 2009; Puente-Palacios et al., 2016), uma vez que está ligada ao indivíduo na organização.

Esclarece-se que, como os objetivos deste estudo não incluem realizar análises sobre o efeito da congruência de valores organizacionais na satisfação no trabalho, mas sim a comparação dos modelos de análise, decidiu-se por não se apresentar extensas informações a respeito dos construtos. Estudos sobre esses temas (VO e ST), que conduzem a outras referências de qualidade, encontram-se em Bowling, Khazon, Meyer e Burrus (2015), Jourdain e Chênevert (2015), Sousa e Porto (2015) e Oliveira e Souza (2014), por exemplo.

\section{Método}

\section{Participantes}

A amostra foi composta por 213 pessoas, oriundas de todas as regiões do Brasil. A idade variou entre 17 e 71 anos $(M=33,40, D P=10,99)$, sendo que $54 \%$ (115) dos participantes eram do sexo masculino. Com relação à escolaridade, 95,3\% (203) disseram possuir nível superior incompleto, completo ou pós-graduação.

Quanto ao porte das organizações, todas do setor privado, $26,8 \%$ (57) dos respondentes disseram trabaIhar em empresas com até 30 funcionários, 41,3\% (88) em empresas com 31 a 100 funcionários, 20,7\% (44) em empresas com 101 a 300 funcionários, 1,4\% (3) de 301 a 500 funcionários e 9,9\% (21) trabalhavam em empresas com mais de 500 funcionários. O tempo de serviço na organização variou entre 0 e 31 anos.

\section{Instrumentos}

Inventário de Valores Organizacionais - IVO (Tamayo et al., 2000). O IVO foi utilizado para medir os valores organizacionais. Com ele, tanto os valores reais, aqueles percebidos pelo funcionário como atualmente praticados pela organização, quanto os valores desejáveis, aqueles que o funcionário entende que deveriam ser praticados pela organização, são pontuados entre 0 e 6, em todos os 36 itens do instrumento. Desta forma, o IVO possibilita a obtenção dos escores de valores reais (EVR) e dos escores de valores desejados (EVD).

Esse instrumento conta com três dimensões bipolares. A primeira é definida pelo eixo autonomia (4 itens, $a=0,83$ ), em oposição ao fator conservadorismo ( 5 itens, $a=0,77$ ). A segunda dimensão é formada pelo fator hierarquia (10 itens, $a=0,87$ ), em oposição ao fator igualitarismo ( 7 itens, $a=0,85$ ). Por fim, na terceira e última dimensão, encontra-se o fator domínio (8 itens, $a=0,84)$ e, em seu polo oposto, o fator harmonia (2 itens, $a=0,85$ ).

Escala de Satisfação no Trabalho - EST (Siqueira, 2008). A EST avalia o grau de satisfação do trabalhador frente a cinco dimensões: o salário; os colegas; a chefia; as promoções; e a natureza do trabalho. Trata-se de uma escala do tipo Likert de 7 pontos. É composta por 25 itens, com cinco itens para cada dimensão. Os coeficientes alfa de Cronbach obtidos para cada fator de satisfação no primeiro estudo que encontrou evidências de validade para a escala foram de 0,86 (com os colegas), 0,92 (com o salário), 0,90 (com a chefia), 0,82 (com a natureza do trabalho) e 0,87 (com as promoções).

\section{Procedimento}

Coleta de dados. Para a coleta de dados foi utilizada uma ferramenta web desenvolvida com base na linguagem Java e utilizando base de dados interna PostgreeSQL, um sistema livre de gerenciamento de bases de dados. A hospedagem foi realizada sobre servidor com segurança de acesso e banco em sistema isolado. Os participantes foram convidados por e-mail ou por redes sociais (Facebook e Linkedin), sendo integrantes da rede de contatos dos pesquisadores e de pessoas próximas. Era requisito estar trabalhando em alguma organização, com qualquer tipo de contrato de trabalho. As respostas poderiam ser fornecidas em qualquer ambiente.

A ferramenta apresentava inicialmente o Termo de Consentimento Livre e Esclarecido (TCLE), previamente aprovado por um comitê de ética (CAAE 15120713.2.0000.5514) e, apenas depois de 
selecionada a opção por participar, os questionários eram apresentados aos respondentes, que poderiam abandonar a pesquisa a qualquer momento, caso desejado. O IVO foi apresentado primeiro e depois a EST. O tempo aproximado de resposta foi de 25 minutos.

Análise de dados. Após a coleta os dados foram exportados para uma base de um pacote de análise estatística. Foram, então, gerados os escores dos valores reais e desejados, os cálculos das diferenças de escores entre eles, os cálculos dos escores de satisfação no trabalho e outros resultados obtidos por meio de estatísticas descritivas e inferenciais, tais como os índices de precisão para os fatores das escalas.

Para conhecer as diferenças de pontuação entre valores reais e desejados foram utilizados dois cálculos, cujos resultados são denominados de Coeficiente de Satisfação com os Valores Organizacionais (CSVO) e Grau de Satisfação com os Valores Organizacionais (GSVO). O CSVO foi obtido por meio das diferenças algébricas entre os valores reais e os valores desejados, conforme recomendado por Tamayo e Borges (2006). Assim, CSVO $_{d}=\left(\sum_{i=x}^{y}(E V D-E V R)\right) / n_{d} . O$ cálculo do GSVO, por sua vez, considerou o valor modular da diferença entre os escores dos valores reais e desejados e utilizou a fórmula GSVO ${ }_{d}=\left(\sum_{i=x}^{y}\left|\left(E V D_{i}-E R_{i}\right)\right|\right) / n_{d}$.

Em ambas as fórmulas d representa a dimensão, EVD o escore obtido para o valor desejado, EVR o escore obtido para o valor real, $x$ é número do primeiro item pertencente à dimensão $\mathrm{d}$, y é o número do último item e n é número de itens na dimensão d. Dessa forma, por usar a função matemática módulo em sua fórmula, o GSVO fornece o resultado das diferenças absolutas entre os valores reais e os desejados. O GSVO foi também utilizado para calcular o Índice de Satisfação com os Valores Organizacionais (ISVO), por meio de uma transformação escalar que possibilitou passar da base 7 (da escala tipo Likert), para a base percentual. Assim, ISVO = $100-\left(\left(\right.\right.$ GSVO $\left.\left._{\mathrm{d}} / 7\right) * 100\right)$.

Dando sequência ao processo, foram realizadas análises de regressão em três etapas. A primeira e a segunda etapas referem-se à execução dos processos de regressão linear múltipla, pelo método ENTER. Primeiramente utilizaram-se os fatores do CSVO como variáveis independentes e as variáveis dependentes foram cada um dos fatores da EST. Posteriormente outra regressão linear múltipla foi realizada com os fatores do GSVO como variáveis independentes e, mais uma vez, as variáveis dependentes foram cada um dos fatores da EST.
Por fim, foi realizada a análise de regressão quadrática na forma como recomendada por Edwards e Parry (1993) e posteriormente detalhada por Shanock, Baran, Gentry, Pattison, e Heggestad (2010). Assim, foram executadas análises de regressão, utilizando a fórmula: $Z=b_{0}+b_{1} X+b_{2} Y+b_{3} X^{2}+b_{4} X Y+b_{5} Y^{2}+e$, onde $Z$ representa os escores para cada um dos fatores da EST, $X$ representa os escores de cada um dos fatores do IVO em sua componente de valores reais, Y representa os escores dos fatores do IVO em sua componente de valores desejados, $\mathrm{b}_{0} \mathrm{a}_{5}$ representam as constantes $\mathrm{da}$ equação e "e" representa o erro. As informações teóricas a respeito desse modelo de regressão e da fórmula utilizada são amplamente apresentadas pelos autores referenciados. Cabe reforçar neste trabalho, entretanto, que $X$ é equivalente a EVR e $Y$ é equivalente a EVD, que foram as notações utilizadas nas fórmulas para o cálculo do coeficiente e do grau de satisfação com os valores organizacionais, i.e. CSVO e GSVO. Desta forma, observa-se que a notação recomendada pelos autores é justamente uma alternativa para se analisar, sob o método da regressão quadrática, a compatibilidade indivíduo-organização dos respondentes com relação aos valores reais e desejados.

Com os resultados obtidos na aplicação da última fórmula apresentada calcularam-se também os valores dos coeficientes $a_{1}$ e $a_{2}$ (inclinação e curvatura da linha de congruência total, $X=Y$ ) e $a_{3}$ e $a_{4}$ (inclinação e curvatura da linha de incongruência total, $X=-Y$ ) para o caso da combinação de fatores a ser usada na análise completa da regressão quadrática. De acordo com Edwards e Parry (1993) e com Shanock et al. (2010), no uso de regressão quadrática esses são os coeficientes que permitem realizar a análise de teste de superfície, diferentemente da regressão linear, que utiliza os coeficientes $\beta$ em sua interpretação.

Em função de valores organizacionais e satisfação no trabalho configurarem-se como medidas multidimensionais, a análise completa da regressão quadrática, incluindo a apresentação de gráficos de superfície será apresentada para os fatores que obtiveram os maiores índices de precisão nas medidas realizadas neste estudo. Isso evitará o acompanhamento de um trabalho extenso, sem prejuízo para a compreensão da análise.

\section{Resultados}

Inicialmente verificaram-se os índices de precisão para os fatores das duas escalas. Foram obtidos os 
Regressão linear e quadrática: Análises comparativas de efeito em medidas do comportamento organizacional

seguintes valores para os fatores do IVO: 0,85 (autonomia), 0,76 (conservadorismo), 0,88 (hierarquia), 0,88 (igualitarismo), 0,82 (domínio) e 0,51 (harmonia). Observa-se que, à exceção do fator harmonia, composto por apenas dois itens, todos os índices de precisão mostraram-se satisfatórios. Em função desses resultados, o fator harmonia foi excluído dos processos de análise. Para a EST foram obtidos os valores $0,86,0,94,0,91,0,85$ e 0,90 para os índices de precisão relativos à satisfação com colegas, ao salário, à chefia, à natureza do trabalho e às promoções.

Prosseguiu-se, então, com a análise das regressões. A Tabela 1 apresenta os resultados das regressões lineares múltiplas.

Tabela 1. Resultados das Regressões Lineares Múltiplas

\begin{tabular}{|c|c|c|c|c|c|}
\hline & \multicolumn{5}{|c|}{ CSVOP } \\
\hline & $R^{2}$ & $R^{2}$ ajustado & $D P$ & $F$ & $p$ \\
\hline Colegas & 0,23 & 0,21 & 0,88 & 12,55 & $<0,001$ \\
\hline Salário & 0,22 & 0,20 & 1,21 & 11,52 & $<0,001$ \\
\hline Chefia & 0,27 & 0,25 & 1,12 & 15,26 & $<0,001$ \\
\hline Natureza & 0,17 & 0,15 & 0,99 & 8,31 & $<0,001$ \\
\hline \multirow[t]{3}{*}{ Promoções } & 0,26 & 0,24 & 1,13 & 14,64 & $<0,001$ \\
\hline & \multicolumn{5}{|c|}{ GSVO } \\
\hline & $R^{2}$ & $R^{2}$ ajustado & $D P$ & $F$ & $p$ \\
\hline Colegas & 0,25 & 0,23 & 0,87 & 13,41 & $<0,001$ \\
\hline Salário & 0,23 & 0,21 & 1,20 & 12,49 & $<0,001$ \\
\hline Chefia & 0,26 & 0,24 & 1,13 & 14,67 & $<0,001$ \\
\hline Natureza & 0,17 & 0,15 & 0,96 & 8,35 & $<0,001$ \\
\hline Promoções & 0,28 & 0,26 & 1,12 & 15,84 & $<0,001$ \\
\hline
\end{tabular}

Os dados da Tabela 1 mostram que todas as regressões múltiplas apresentaram resultados estatisticamente significativos ao nível $p<0,001$. Os valores de $R 2$ ajustado mostraram-se muito próximos, em todos os casos, nas regressões aplicadas com os fatores de CSVO e nas aplicadas com os fatores de GSVO.

A Tabela 2 apresenta os resultados das regressões quadráticas. As comparações entre os modelos consideram em conjunto os dados apresentados na Tabela $1 \mathrm{e}$ na Tabela 2.

Tomando-se a variável salário como dependente, observou-se $\Delta R 2=0,20$ quando CSVO é utilizado como variável independente e $\Delta R 2=0,21$ para GSVO. Em ambos os casos, os resultados são menores do que a variância explicada para a satisfação com o salário na aplicação da regressão quadrática com os fatores autonomia e igualitarismo solitariamente, o que indica que o modelo quadrático mostrou maior potencial de explicação quando comparado ao modelo de RL múltipla.
Tabela 2. Resultados das Regressões Quadráticas

\begin{tabular}{|c|c|c|c|c|}
\hline \multirow{3}{*}{ EST } & \multirow{3}{*}{ IVO } & \multicolumn{3}{|c|}{ Regressão quadrática } \\
\hline & & \multicolumn{3}{|c|}{ Polinômio } \\
\hline & & $R^{2}$ ajustado & $D P$ & $p$ \\
\hline \multirow{5}{*}{$\begin{array}{l}\frac{n}{0} \\
\frac{0}{0} \\
0 \\
\end{array}$} & Autonomia & 0,05 & 0,965 & 0,012 \\
\hline & Conservadorismo & 0,28 & 0,839 & $<0,001$ \\
\hline & Hierarquia & 0,08 & 0,947 & $<0,001$ \\
\hline & Igualitarismo & 0,11 & 0,932 & $<0,001$ \\
\hline & Domínio & 0,09 & 0,943 & $<0,001$ \\
\hline \multirow{5}{*}{$\frac{\circ}{\frac{0}{\frac{\pi}{\pi}}}$} & Autonomia & 0,24 & 1,180 & $<0,001$ \\
\hline & Conservadorismo & 0,12 & 1,269 & $<0,001$ \\
\hline & Hierarquia & 0,12 & 1,275 & $<0,001$ \\
\hline & Igualitarismo & 0,26 & 1,167 & $<0,001$ \\
\hline & Domínio & 0,14 & 1,258 & $<0,001$ \\
\hline \multirow{5}{*}{$\begin{array}{l}\frac{\pi}{\mathbb{2}} \\
\frac{\pi}{U}\end{array}$} & Autonomia & 0,24 & 1,128 & $<0,001$ \\
\hline & Conservadorismo & 0,17 & 1,178 & $<0,001$ \\
\hline & Hierarquia & 0,20 & 1,161 & $<0,001$ \\
\hline & Igualitarismo & 0,25 & 1,118 & $<0,001$ \\
\hline & Domínio & 0,15 & 1,192 & $<0,001$ \\
\hline \multirow{5}{*}{ 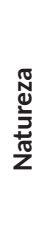 } & Autonomia & 0,15 & 0,959 & $<0,001$ \\
\hline & Conservadorismo & 0,09 & 0,989 & $<0,001$ \\
\hline & Hierarquia & 0,11 & 0,980 & $<0,001$ \\
\hline & Igualitarismo & 0,18 & 0,940 & $<0,001$ \\
\hline & Domínio & 0,15 & 0,958 & $<0,001$ \\
\hline \multirow{5}{*}{ 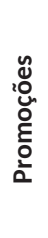 } & Autonomia & 0,28 & 1,097 & $<0,001$ \\
\hline & Conservadorismo & 0,16 & 1,192 & $<0,001$ \\
\hline & Hierarquia & 0,08 & 1,244 & $<0,001$ \\
\hline & Igualitarismo & 0,30 & 1,084 & $<0,001$ \\
\hline & Domínio & 0,13 & 1,210 & $<0,001$ \\
\hline
\end{tabular}

Com relação aos coeficientes, para a regressão realizada com os fatores do CSVO, os resultados encontrados foram $\beta=0,42(p \leq 0,001)$ na regressão do fator autonomia e $\beta=0,27(p<0,05)$ na regressão para o fator igualitarismo. Os demais coeficientes não apresentaram significância estatística. Por sua vez, os resultados encontrados na regressão realizada com os fatores do GSVO foram $\beta=-0,46$ $(p<0,001)$ para o fator autonomia, sendo que os demais coeficientes não apresentaram significância estatística.

Para realizar a análise completa do modelo de regressão quadrática conforme critério explicitado (alfa de Cronbach), foi selecionado o fator salário da EST como variável dependente e os fatores hierarquia e igualitarismo do IVO, separadamente, como variáveis independentes. A Tabela 3 mostra os resultados obtidos, em complementação aos já apresentados na Tabela 2, e as figuras 1 e 2 apresentam os gráficos para análise de superfície. 
Tabela 3. Análise de Superfície - Covariâncias e Coeficientes da Regressão Quadrática,de Inclinação e de Curvatura nos Planos.

\begin{tabular}{|c|c|c|c|}
\hline \multirow{2}{*}{$\begin{array}{l}\text { EST } \\
\text { IVO }\end{array}$} & & \multicolumn{2}{|c|}{ Salário } \\
\hline & & Hierarquia & Igualitarismo \\
\hline \multirow[t]{2}{*}{ Intercepto } & $\mathrm{b}_{0}$ & $3,58^{*}$ & $4,03^{*}$ \\
\hline & $D P$ & 0,21 & 0,44 \\
\hline \multirow[t]{2}{*}{$\mathrm{X}$ (valor real) } & $b_{1}$ & $-0,15$ & 0,45 \\
\hline & $D P$ & 0,31 & 0,27 \\
\hline \multirow[t]{2}{*}{ Y (valor desejado) } & $\mathrm{b}_{2}$ & 0,09 & $-0,51$ \\
\hline & $D P$ & 0,29 & 0,49 \\
\hline \multirow[t]{2}{*}{ XY (real x desejado) } & $\mathrm{b}_{3}$ & 0,26 & 0,06 \\
\hline & $D P$ & 0,14 & 0,12 \\
\hline \multirow[t]{2}{*}{$\mathrm{X}^{2}\left(\right.$ valor real) ${ }^{2}$} & $\mathrm{b}_{4}$ & $-0,01$ & 0,02 \\
\hline & $D P$ & 0,08 & 0,06 \\
\hline \multirow[t]{2}{*}{$\mathrm{Y}^{2}$ (valor desejado) ${ }^{2}$} & $\mathrm{~b}_{5}$ & $-0,05$ & 0,15 \\
\hline & $D P$ & 0,10 & 0,14 \\
\hline \multirow[t]{4}{*}{ Covariâncias } & $b_{1} b_{2}$ & $-0,057$ & $-0,084$ \\
\hline & $\mathrm{b}_{3} \mathrm{~b}_{4}$ & $-0,003$ & $-0,003$ \\
\hline & $\mathrm{b}_{3} \mathrm{~b}_{5}$ & $-0,001$ & $-0,001$ \\
\hline & $b_{4} b_{5}$ & $-0,008$ & $-0,008$ \\
\hline \multirow[t]{4}{*}{ Inclinação e curvatura } & $a_{1}$ & $-0,06$ & $-0,06$ \\
\hline & $a_{2}$ & $0,20^{* *}$ & $0,23^{* *}$ \\
\hline & $a_{3}$ & $-0,24$ & 0,96 \\
\hline & $a_{4}$ & $-0,32$ & 0,11 \\
\hline
\end{tabular}

nota: ${ }^{*} p<0,001,{ }^{* *} p<0,05$

Observa-se na Tabela 3, que foram encontrados valores com significância estatística para o coeficiente $a_{2}$ nos dois casos analisados, isto é, tanto na regressão quadrática do valor (real e desejado) hierarquia, quanto na regressão quadrática do valor igualitarismo (real e desejado), tendo a satisfação com o salário como variável dependente. A significância estatística encontrada para esses coeficientes indica um relacionamento não linear entre as variáveis independentes e a variável dependente ao longo de linha de congruência (Shanock et al., 2010), o que implica que o modelo quadrático mostrou-se também capaz de elucidar mais informações a respeito das relações entre as variáveis. Os demais coeficientes $\left(a_{1}, a_{3}\right.$ e $\left.a_{4}\right)$ não apresentaram valores estatisticamente significativos.

O gráfico para análise de superfície apresentado na Figura 1 permite observar que a satisfação com o salário aumenta na medida em que existe uma congruência entre os valores reais e desejados para o fator hierarquia. Esse aumento se dá de forma ligeiramente mais acentuada quando as pontuações para os valores (real e desejado) são mais baixas, do que quando são mais altas. O gráfico em forma de sela leva ao entendimento de que a satisfação com o salário decresce quando existe incongruência entre os valores reais e desejados, em ambas as direções da superfície ao longo da linha de incongruência perfeita. No entanto, como os testes para os coeficientes $a_{3}$ e $a_{4}$ não indicaram significância estatística (Tabela 3), apenas a curva relativa à congruência pode ser analisada com segurança.

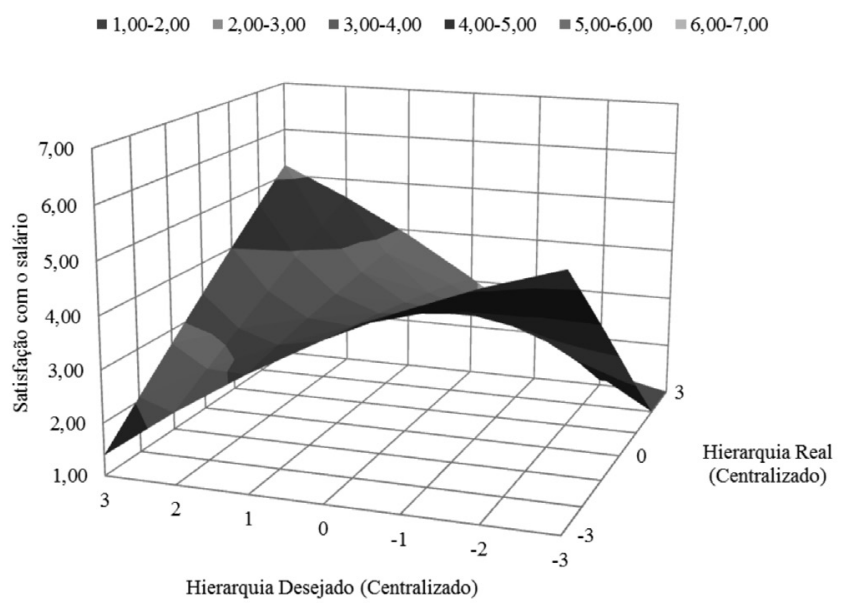

Figura 1. Impacto dos Valores Reais e Desejados (Fator Hierarquia) na Satisfação com o Salário.

Também o gráfico da Figura 2 permite avaliar que a satisfação com o salário cresce não linearmente na medida em que existe congruência entre o valor igualitarismo real e desejado, sendo esse crescimento ligeiramente mais acentuado quando igualitarismo real e desejado recebem pontuações mais baixas do que no sentido em que pontuam mais alto. Mais uma vez, dado que apenas o coeficiente $a_{2}$ apresentou significância estatística, a curva relativa à linha de incongruência perfeita não pode ser interpretada com segurança. Ela revela, porém, que a satisfação com o salário diminui quando a medida real para o valor igualitarismo é menor que a medida da expectativa a respeito desse valor e aumenta quando a medida real é maior do que a da expectativa.

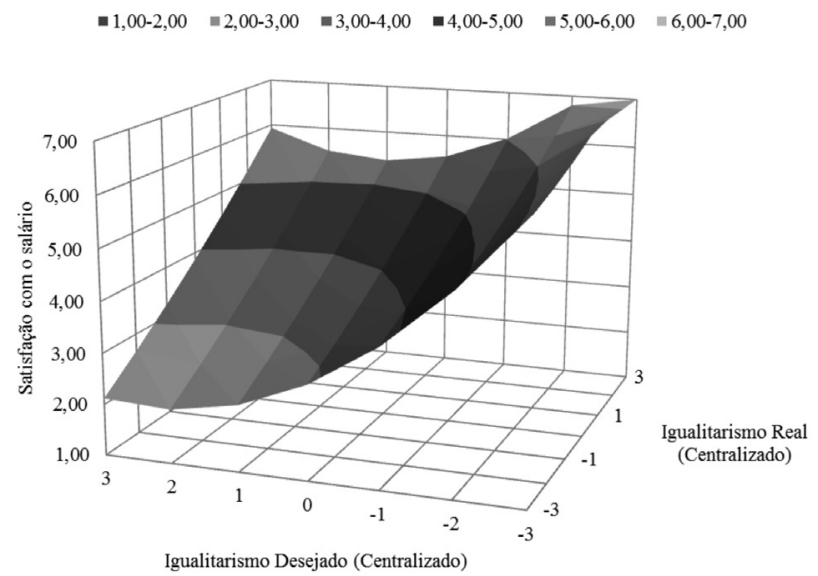

Figura 2. Impacto dos Valores Reais e Desejados (Fator Igualitarismo) na Satisfação com o Salário. 
Regressão linear e quadrática: Análises comparativas de efeito em medidas do comportamento organizacional

\section{Discussão}

Utilizando diferentes modelos de análise de regressão estatística foi possível constatar diferenças entre suas capacidades de explicação do efeito da combinação de duas variáveis independentes sobre uma variável dependente. Foram utilizadas as variáveis valores organizacionais reais e desejados como independentes, e a variável satisfação com o trabalho como dependente. Os métodos de análise aplicados, regressão linear múltipla e regressão quadrática, utilizam as variáveis independentes de forma diferente, colapsando-as por meio de um índice no caso do estudo dos modelos lineares (CSVO e GSVO, neste estudo), ou agregando-as sob a forma de uma equação polinomial de segundo grau no modelo quadrático.

Os resultados permitiram verificar que a regressão quadrática possibilitou encontrar explicações de variância de maior magnitude, quando comparada aos modelos lineares. A análise de gráficos de superfície viabilizada por esse modelo também é capaz de ampliar o entendimento a respeito dos efeitos promovidos pelas variáveis independentes sobre as dependentes. Esses achados estão de acordo com o encontrado na literatura, conforme modelo adotado ou defendido por diversos autores (De Haas \& Van Eerde, 2015; Edwards, 1994a, 1994b; Edwards \& Parry, 1993; Lee \& Antonakis, 2014; Ramsey \& van den Berk-Clark, 2015; Shanock et al., 2010; Sousa \& Porto, 2015).

Achados como o deste estudo são alguns dos motivos que levaram Edwards (1994a, 2008) a defender que estudiosos não apenas apliquem a solução quadrática para testar teorias de POF em suas pesquisas, mas também que teóricos pensem e formulem suas teorias, postulando-as de maneira a não conduzir o pesquisador ao engano de testá-las por meio de métodos menos adequados, como o método baseado em diferenças de pontuação. De acordo com Edwards (2008), muitos pesquisadores são levados a colapsar variáveis em um único índice, como os casos de CSVO e GSVO, porque as teorias que tratam de compatibilidade indivíduo-organização - POF - conduzem a esse caminho, afirmando, por exemplo, que é a POF que está relacionada a um resultado, ao invés de afirmar que pessoa, organização e suas interações estão relacionadas a ele.

É assim que, conforme Edwards (1994a), pesquisadores devem levar todos esses pontos em consideração e testar os modelos teóricos que estudam utilizando o método que possibilite a análise mais ampla a respeito dos construtos envolvidos, ou ainda, devem aplicar diversos modelos viáveis para selecionar o mais adequado entre eles. Neste sentido, é relevante considerar que comparações entre métodos de análise não devem se restringir apenas a diferentes tipos de regressão, como os apresentados neste trabalho. É de importância que o pesquisador fortaleça seu modelo teórico estabelecendo com clareza a que nível (micro, meso, macro) pertencem as variáveis presentes em seus estudos do comportamento organizacional e amplie a testagem em busca do método mais adequado de análise, por exemplo, aplicando e avaliando também resultados de análises multinível (Puente-Palacios \& Laros, 2009; Puente-Palacios et al., 2016).

Esta é, entretanto, uma conclusão à qual se pode chegar quando se tratam de análises de cunho acadêmico. Esse é o caso do caminho traçado neste estudo, que trata de POF como uma subárea de estudos de medidas do comportamento organizacional, que constituem uma parcela de estudos de PO\&T. Porém, como lembrado por Borges-Andrade e Pagotto (2010), a PO\&T passa por processos de desenvolvimento relacionados tanto à pesquisa científica, quanto à atuação na prática organizacional, devendo, assim, permitir que o avanço do conhecimento seja aplicado no cotidiano das empresas.

Assim, para a aplicação cotidiana na prática organizacional, recorda-se que o IVO é um instrumento desenvolvido não apenas com o intuito de medir valores organizacionais, mas também as diferenças de pontuação entre os valores reais e desejados de forma a compreender o nível de satisfação/insatisfação de colaboradores organizacionais (Tamayo \& Borges, 2006; Tamayo et al., 2000). Recorda-se também que, para atender aos critérios da FNQ (2008), um indicador de gestão deve ser de fácil visibilidade, possibilitar uma visão balanceada do desempenho da organização, facilitar o entendimento dos direcionadores do negócio, e suportar a tomada de decisões visando influenciar os ambientes interno e externo à organização. Entende-se daí que, na prática organizacional, quanto mais simples for a compreensão e a comunicação de uma medida, maior será a propensão para sua adoção.

Desta forma, dado o contexto organizacional e os resultados encontrados por meio deste estudo, sugere-se que, uma vez que todos os modelos de regressão aplicados indicaram que as medidas de satisfação com o trabalho sofrem impacto com alterações que ocorrem nas medidas de congruência de valores, o profissional 
da prática poderá, se for mais adequado ao seu contexto e aos recursos disponíveis, considerar a construção de suas metas e de projetos de melhorias de gestão tomando por base as expectativas visíveis por meio da aplicação das diferenças de pontuação e da regressão linear, o que é, de certa forma, aderente à visão defendida por Tisak e Smith (1994). Essas expectativas são mostraram-se, nesta comparação, coerentes com os resultados da regressão quadrática e, por sua vez, possibilitam explicações mais aderentes ao esperado na definição de indicadores de gestão, conforme FNQ (2008).

Ainda, a despeito dos cálculos definidos por Tamayo e Borges (2006) que levam aos resultados do CSVO, sugere-se também que o cálculo das diferenças de pontuação seja realizado por meio da diferença absoluta. Essa é a forma de cálculo que permite transformar os resultados do coeficiente de satisfação (GSVO) em um índice de satisfação percentual (ISVO), também mais aderente aos requisitos para adoção de um indicador de gestão (FNQ, 2008).

Por fim, sugere-se que o racional apresentado neste estudo é facilmente transponível para aplicações e comparações a serem realizadas com outros instrumentos e outros conjuntos de variáveis independentes e dependentes, abrangidos pelo contexto de estudos de POF. Desta forma, tanto profissionais ligados à pesquisa acadêmica, quanto os ligados à prática organizacional podem se beneficiar dessas análises e considerações, transpondo-as para suas pesquisas e organizações de acordo com suas necessidades cotidianas.

Naturalmente, este estudo apresenta limitações. Uma delas está ligada à apresentação exclusiva de apenas dois dos gráficos de análise de superfície no caso do modelo de regressão quadrática. Para reduzir essa limitação, os autores poderão enviar todos os gráficos a quem tiver interesse, mediante solicitação para a autora de contato. O estudo é também limitado em sua extensão de análise matemática, que poderia ser ampliada por meio da apresentação de cálculos estatísticos a respeito das restrições aplicadas às variáveis, conforme cada modelo de regressão aplicado. Entretanto, estudos extensivos a esse respeito estão disponíveis na literatura internacional e este trabalho não teria novas contribuições a agregar nesse sentido. Sugere-se, portanto, a visita direta às fontes, em especial Edwards e Parry (1993), Edwards (1994a, 1994b) e Shanock et al. (2010).

Existe, ainda, uma limitação relativa à qualidade dos dados coletados. A forma de aplicação do IVO, de acordo com Tamayo et al. (2000), em que se apresentam, lado a lado, as tabelas para pontuação de valores reais e de valores desejáveis poderia, eventualmente, provocar um viés nas respostas por motivar a interpretação de que o desejado normalmente deveria ser "algo a mais" do que o que já se tem. Se esse viés existe, então não apenas os coeficientes e o índice de satisfação/insatisfação carregam reflexos dele, mas também o conjunto das variáveis adotadas na regressão quadrática. Esse tipo de viés relativo à coleta de dados em um único momento é amplamente discutido na literatura, a exemplo de Podsakoff, MacKenzie, Lee e Podsakoff (2003). Novos estudos poderão ser realizados utilizando diferentes sequências, ou momentos, de apresentação das escalas. Isso seria contributivo para o alcance de conclusões mais precisas sobre o tema.

Uma segunda limitação relativa à qualidade dos dados coletados diz respeito ao fato de a coleta ter sido realizada em momento único, não permitindo testar o ajuste do modelo quadrático ao longo do tempo. A realização de novas pesquisas de cunho longitudinal seria também contributiva para agregar informações a respeito do ajuste do modelo.

\section{Referências}

Borges-Andrade, J. E., \& Pagotto, C. P. (2010). O estado da arte da pesquisa Brasileira em Psicologia do Trabalho e Organizacional. Psicologia: Teoria e Pesquisa, 26, 37-50. Recuperado de http://periodicos.unb.br/index.php/revistaptp/article/download/20709/14760

Borges, L. D. O., Argolo, J. C. T., \& Baker, M. C. S. (2006). Os valores organizacionais e a síndrome de burnout: dois momentos em uma maternidade pública. Psicologia: Reflexão e Crítica, 19(1), 34-43. Recuperado de http://www.redalyc.org/html/188/18819106/

Borges, L. D. O., \& Barros, S. C. (2015). Inventário de significado do trabalho para trabalhadores de baixa instrução. In K. Puente-Palácios \& A. L. A. Peixoto (Orgs.), Ferramentas de diagnóstico para organizações e trabalho: um olhar a partir da psicologia (pp. 232-260). Porto Alegre: Artmed.

Bowling, N. A., Khazon, S., Meyer, R. D., \& Burrus, C. J. (2015). Situational strength as a moderator of the relationship between job satisfaction and job performance: a meta-analytic examination. Journal of Business and Psychology, 30(1), 89-104. doi: 10.1007/ s10869-013-9340-7

De Haas, M. J., \& Van Eerde, W. (2015). Archetypes and person-organization fit in law firms. Journal of Professions and Organization, 0 , 1-19. doi: 10.1093/jpo/jov003

Edwards, J. R. (1994a). The study of congruence in organizational behavior research: critique and a proposed alternative. Organizational Behavior and Human Decision Processes, 58, 51-100. doi: 10.1006/ obhd.1994.1029

Edwards, J. R. (1994b). Regression analysis as an alternative to difference scores. Journal of Management, 20(3), 683-689. doi: $10.1177 / 014920639402000311$ 
Regressão linear e quadrática: Análises comparativas de efeito em medidas do comportamento organizacional

Edwards, J. R. (2001). Ten difference score myths. Organizational Research Methods, 4(3), 265-287. doi: 10.1177/109442810143005

Edwards, J. R. (2008). Person-environment fit in organizations: an assessment of theoretical

Progress. The Academy of Management Annals, 2, 167-230. doi: 10.1080/19416520802211503

Edwards, J. R., \& Parry, M. E. (1993). On the use of polynomial regression equations as an alternative to difference scores in organizational research. Academy of Management Journal, 36, 1577-1613. doi: $10.2307 / 256822$

Floyd, S. W., \& Sputtek, R. (2011). Rediscovering the individual in strategy: methodological challenges, strategies, and prospects. In D. J. Ketchen Jr. \& D. D. Bergh (Orgs), Building Methodological Bridges. Research Methodology in Strategy and Management (pp. 3-30). Bingley: Emerald Group Publishing. doi: 10.1108/ s1479-8387(2011)0000006004

Fundação Nacional da Qualidade (2008). Cadernos Compromisso Com a Excelência - Resultados. São Paulo: Fundação Nacional da Qualidade.

Jourdain, G., \& Chênevert, D. (2015). The moderating influence of perceived organizational values on the burnout-absenteeism relationship. Journal of Business and Psychology, 30(1), 177-191. doi: 10.1007/s10869-014-9346-9

Kristof, A. L. (1996). Person? ?organization fit: an integrative review of its conceptualizations, measurement, and implications. Personnel Psychology, 49(1), 1-49. Recuperado de http://leeds-faculty.colorado.edu/dahe7472/kristoff1996.pdf

Lee, Y.T., \& Antonakis, J. (2014). When preference is not satisfied but the individual is how power distance moderates person-job fit. Journal of Management, 40(3), 641-675. doi: 10.1177/0149206311436080

Oliveira, A. D. F., \& Souza, M. A. D. (2014). Confiança do empregado na organização: o impacto dos valores pessoais e organizacionais. Revista Psicologia Organizações e Trabalho, 14(2), 204-217. Recuperado de http://pepsic.bvsalud.org/scielo. php?script=sci_arttext\&pid=S1984-66572014000200007

Podsakoff, P. M., MacKenzie, S. B., Lee, J. Y., \& Podsakoff, N. P. (2003). Common method biases in behavioral research: a critical review of the literature and recommended remedies. Journal of Applied Psychology, 88(5), 879-903. doi: 10.1037/0021-9010.88.5.879
Porto, J. B., Tamayo, M., \& Paschoal, T. (2012). (In)congruência pessoa-ambiente: relações com bem-estar, estresse e burnout. In M. C. Ferreira \& H. Mendonça (Orgs.), Saúde e bem-estar no trabalho: dimensões individuais e culturais (pp. 229-259). São Paulo, SP: Casa do Psicólogo.

Puente-Palacios, K. E., \& Laros, J. A. (2009). Análise multinível: contribuições para estudos sobre efeito do contexto social no comportamento individual. Estudos de Psicologia, 26, 349-61. Recuperado de http://www.scielo.br/pdf/estpsi/v26n3/v26n3a08

Puente-Palacios, K. E., Porto, J. B., \& Martins, M. D. C. F. (2016). A emersão na articulação de níveis em Psicologia Organizacional e do Trabalho. Revista Psicologia Organizações e Trabalho, 16(4), 358-366. doi: 10.17652/rpot/2016.4.12603

Ramsey, A. T., \& van den Berk-Clark, C. (2015). Provider-agency fit in substance abuse treatment organizations: implications for learning climate, morale, and evidence-based practice implementation. BMC Research Notes, 8(1), 194-204. doi: 10.1186/s13104-015-1110-3

Saraç, M., Efil, I., \& Eryilmaz, M. (2014). A study of the relationship between person-organization fit and employee creativity. Management Research Review, 37(5), 479-501. doi: 10.1108/MRR-01-2013-0025

Shanock, L. R., Baran, B. E., Gentry, W. A., Pattison, S. C., \& Heggestad, E. D. (2010). Polynomial regression with response surface analysis: a powerful approach for examining moderation and overcoming limitations of difference scores. Journal of Business and Psychology, 25(4), 543-554. doi: 10.1007/s10869-010-9183-4

Siqueira, M. M. M. (2008). Satisfação no trabalho. In M. M. M. Siqueira e cols (Orgs.). Medidas do comportamento organizacional (pp. 265274). Porto Alegre: Artmed.

Sousa, J. M. D., \& Porto, J. B. (2015). Happiness at work: organizational values and person-organization fit impact. Paidéia, 25(61), 211-220. doi: 10.1590/1982-43272561201509

Tamayo, A., \& Borges, L. D. O. (2006). Valores do trabalho e das organizações. In M. Ros \& V. Gouveia (Orgs.), Psicologia social dos valores humanos: desenvolvimentos teóricos, metodológicos e aplicados (2a.ed., pp. 397-431). São Paulo: Senac.

Tamayo, A., Mendes A., \& Paz M. G. T. (2000). Inventário de valores organizacionais. Estudos de Psicologia, 5(2), 289-315. doi: 10.1590/S1413-294X2000000200002

Tisak, J., \& Smith, C. S. (1994). Defending and extending difference score methods. Journal of Management, 20, 675-682. doi: $10.1177 / 014920639402000310$

Maria Isabel de Campos, Mestre em Psicologia pela Universidade São Francisco (USF), é Consultora na isabelcampos.com.br. Endereço para correspondência: Rua José Pugliesi Filho, 306 - Ch. Sta. Margarida. CEP 13085-415 - Campinas (SP). Fone: 1932790768.

E-mail: belcampos@uol.com.br

Fabián J. M. Rueda, Doutor em Psicologia pela Universidade São Francisco (USF), é Coordenador do Programa de Pós-Graduação Stricto Sensu em Psicologia da Universidade São Francisco (USF). E-mail: fabian.rueda@usf.edu.br

Recebido em 03.Mar.16 Revisado em 26.Mar.17 Aceito em 03.Mai.17 